\title{
CDISC Vaccines Therapeutic Area User Guide Version 1.1
}

National Cancer Institute

\section{Source}

National Cancer Institute. CDISC Vaccines Therapeutic Area User Guide Version 1.1. NCI

Thesaurus. Code C161455.

The 1.1 version of the CDISC Vaccines therapeutic area user guide. 